\title{
Medicamentos na amamentação: quais as evidências?
}

\author{
Medications in breastfeeding: what evidence is there?
}

Michele Raminelli ${ }^{1}$

Siomara Regina Hahn ${ }^{1}$
${ }^{1}$ Curso de Farmácia, Universidade de Passo Fundo. Br 285 Km 17 Campus I, São José. 99052-900 Passo Fundo RS Brasil.michele_raminelli@ hotmail.com

\begin{abstract}
Breastfeeding plays a fundamental role in the benefits for the health of the newborn child and the nursing mother. The use of medications during breastfeeding is a relevant issue, by virtue of the frequent need for pharmacological treatment in the postpartum period. The scope of this article was to conduct a review of the literature regarding the efficacy and safety of medications used during the breastfeeding period. A search was conducted in the PubMed (National Library of Medicine), ScienceDirect and Biblioteca Virtual em Saúde (BVS) databases for articles published in Portuguese, English and Spanish in the period from 1981 to 2016. This review discusses the risk of the use of medications during lactation and the effects that they may have on the breastfed infant. Few medications are contraindicated and others require care due the risk of adverse effects on breastfed infants or in the suppression of breast milk volume. Therefore, the dissemination of updated information for the health professional to adequately assess the risks and the benefits of the use of medications during breastfeeding is of vital importance, thereby contributing to avoid early weaning.
\end{abstract}

Key words Medications, lactation, toxicity
Resumo A amamentação desempenha um papel fundamental nos beneficios para a saúde do recém-nascido e da nutriz. O uso de fármacos durante a amamentação é um tema relevante, visto a frequente necessidade de tratamento farmacológico no período pós-parto. O presente artigo teve como objetivo realizar revisão bibliográfica sobre a eficácia e a segurança dos medicamentos utilizados durante o período de amamentação. Foram consultadas as bases de dados PubMed (National Library of Medicine), Science Direct e Biblioteca Virtual em Saúde (BVS), nas línguas portuguesa, inglesa e espanhola, em artigos publicados no período de 1981-2016. Esta revisão discute o risco do uso de medicamentos na lactação e os efeitos que podem causar no lactente. Poucos fármacos são contraindicados e outros requerem cuidados devido ao risco de efeitos adversos em lactentes ou ainda na supressão do volume de leite materno. Portanto, a divulgação de informações atualizadas para o profissional de saúde avaliar adequadamente os riscos e os benefícios do uso de fármacos durante a amamentação é de vital importância e, dessa forma, contribui para evitar o desmame precoce.

Palavras-chave Medicamentos, Lactação, Toxicidade 


\section{Introdução}

A amamentação é uma forma inigualável de proporcionar a nutrição ideal para o desenvolvimento e crescimento saudável dos recém-nascidos. A Organização Mundial da Saúde recomenda a amamentação exclusiva nos primeiros 6 meses de vida e sua continuidade junto com a alimentação complementar até os 2 anos de idade ou mais ${ }^{1}$.

O desenvolvimento da amamentação a um nível praticamente mundial poderia evitar 20.000 mortes anuais por câncer de mama em mulheres, além de 823.000 mortes anuais de crianças menores de 5 anos. Em países de baixa e média renda, somente $37 \%$ das crianças com menos de 6 meses de idade são amamentadas exclusivamente com leite materno ${ }^{2}$.

Em situações em que a nutriz está em processo de terapia farmacológica deve-se equilibrar os benefícios para criança e a mãe contra o risco potencial de exposição ao fármaco para a criança ${ }^{3}$. Muitas mulheres são aconselhadas a interromper a amamentação em virtude do uso de algum medicamento, em razão de preocupações relacionadas a efeitos adversos em seus filhos ${ }^{4}$.

Essas recomendações geralmente não são baseadas em evidências, pois muitas informações não se encontram disponíveis quanto à extensão da excreção do fármaco no leite humano, e para outros fármacos, a informação é limitada aos dados de estudos com animais, que podem não se correlacionar com a experiência humana. Além disso, nem todos os fármacos são excretados no leite materno em quantidades clinicamente significativas ${ }^{5}$.

Embora a maioria dos medicamentos sejam considerados compatíveis com a amamentação, os casos de toxicidade infantil existem, sugerindo avaliação de risco caso a caso antes que a nutriz inicie a amamentação ou terapia medicamentosa ${ }^{6}$.

Torna-se fundamental, portanto, a constante atualização sobre a segurança dos fármacos utilizados na amamentação como instrumento de apoio à prática clínica. Desta forma, o presente artigo teve como objetivo realizar revisão bibliográfica sobre as evidências de eficácia e segurança dos medicamentos utilizados durante o período de amamentação.

\section{Métodos}

O presente estudo trata-se de uma revisão da literatura realizada a partir de buscas nas bases de dados PubMed (National Library of Medici- ne), Science Direct e Biblioteca Virtual em Saúde (BVS), nas línguas portuguesa, inglesa e espanhola, utilizando os descritores "medicamentos", "drogas", "amamentação", "leite materno" e "lactação" em artigos publicados no período de 1981-2016. Os artigos não foram limitados quanto ao seu delineamento. Foram incluídos 80 artigos na revisão final e 13 publicações encontradas em livros e sites específicos sobre o tema. Dos artigos incluídos 75 foram na base de dados Pubmed, 3 na Science Direct e 2 na BVS.

Os estudos selecionados foram aqueles com dados relevantes sobre a excreção de fármacos no leite materno e seus possíveis efeitos no lactente. Outras referências importantes encontradas em livros foram incluídas, a fim de fornecer subsídios para a realização deste artigo. Também foram coletados dados disponíveis sobre o uso de fármacos durante a amamentação na versão eletrônica do livro "Medications and Mother's Milk" de Hale e Rowe ${ }^{7}$ e no banco de dados LactMed $^{8}$ (U.S. National Library of Medicine, 2016) disponível em http://toxnet.nlm.nih.gov/newtoxnet/lactmed.htm. As etapas de inclusão e exclusão dos estudos estão demonstradas conforme Figura 1.

\section{Resultados e discussão}

\section{O uso de medicamentos e amamentação}

Alguns fatores contribuem para que a nutriz opte por não amamentar, entre eles fatores psi-

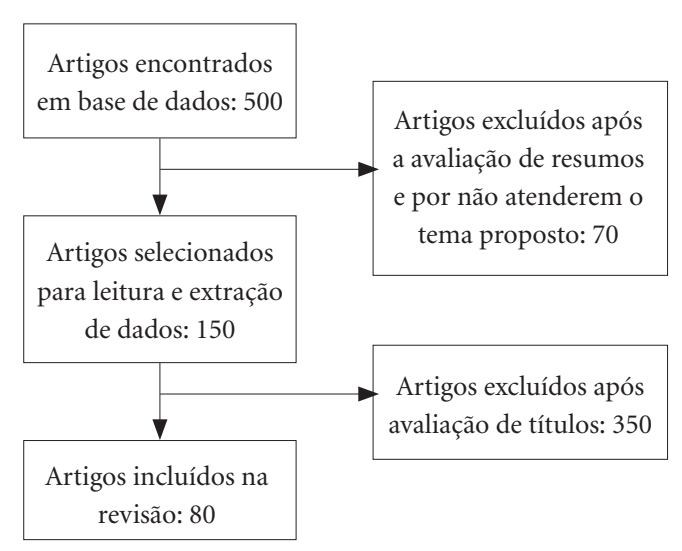

Figura 1. Fluxograma de tratamento para as etapas de inclusão e exclusão dos estudos. 
cológicos, leite materno insuficiente, retorno ao trabalho, estilo de vida e, principalmente, a insegurança quanto à nutrição e peso da criança, bem como se o uso de medicamentos pode afetar negativamente a criança amamentada.

O uso de medicamentos durante a lactação é uma prática frequente ${ }^{9}$, pois muitas vezes há a necessidade de tratamento no período pós-parto para combater problemas de saúde frequentes nesse período como mastite, infecções e até mesmo transtornos depressivos ${ }^{10}$.

Em Caucaia-Ceará, Brasil, estudo quantitativo, com 132 nutrizes de uma unidade básica de saúde registrou que $80 \%$ das nutrizes usaram medicamentos ao longo da amamentação e $58,3 \%$ relataram não ter recebido orientações ${ }^{11}$. Estudo transversal, quantitativo e descritivo realizado com 100 nutrizes no Hospital Municipal de Duque de Caxias-RJ, demonstrou que 78\% faziam uso de algum tipo de medicamento. Das nutrizes em terapia medicamentosa, 76,92\% tiveram orientação durante o tratamento, e todos os medicamentos prescritos foram compatíveis com a amamentação ${ }^{12}$.

Acredita-se que o uso de medicamentos pelas nutrizes é um dos fatores responsáveis pela interrupção precoce do aleitamento materno ${ }^{13}$. No estudo longitudinal de Odom et al. ${ }^{14}$ realizado com 1177 mães de idade igual ou superior a 18 anos, os achados demonstraram que aproximadamente $60 \%$ das que pararam de amamentar não realizaram o tempo de aleitamento preconizado, sendo que a doença ou a necessidade de utilizar medicamento foi um dos fatores de não cumprir o tempo de amamentação desejado. Em estudo retrospectivo, Olang et al. ${ }^{15}$ observaram que $10 \%$ das nutrizes citaram o uso de medicamentos ou doença materna entre as razões para descontinuar a amamentação após os 6 meses de idade do lactente.

\section{Aleitamento materno e seus efeitos na mãe e no lactente}

O leite materno é considerado a nutrição ideal para os recém-nascidos por apresentar uma ampla gama de compostos bioativos e nutrientes essenciais ${ }^{16}$, assim como outras substâncias entre elas hormônios, fatores de crescimento, oligossacarídeos e compostos relacionados ao sistema imunológico, que desempenham um papel importante nos benefícios sobre a criança associados com a amamentação ${ }^{17}$.

Na última década, houve uma evolução significativa dos índices de aleitamento materno no
Brasil. Em pesquisa de corte transversal realizada para apresentar os indicadores de aleitamento materno (AM), denominada de II Pesquisa de Prevalência de Aleitamento Materno nas Capitais Brasileiras e Distrito Federal, observou-se que a prevalência do aleitamento materno exclusivo foi de $41 \%$ em crianças menores de 6 meses, sendo que a Região Norte foi a que apresentou maior prevalência $(45,9 \%)$ e a Região Nordeste apresentou a pior situação (37,0\%). Já para as crianças de 9 a 12 meses a prevalência do aleitamento materno foi de 58,7\%, sendo que a Região Norte apresentou melhor situação $(76,9 \%)$ e a Região Sul apresentou a pior situação $(49,5 \%)^{18}$.

$\mathrm{Na}$ primeira hora do nascimento é fornecido o leite materno para a criança, denominado como primeiro leite ou colostro ${ }^{19}$. Este é produzido de 0 a 3 dias após o parto e é rico em carboidratos, proteínas e anticorpos que auxiliam o recém-nascido a se manter saudável, além de atuar como um laxante para o lactente ${ }^{20}$. Aos 7 e 10 dias após o parto, a mãe começa a produzir o denominado leite maduro, que é composto de grande parte de água, sendo essencial para manter a hidratação do lactente ${ }^{21}$.

Os benefícios da amamentação a curto e longo prazo são documentados. O leite materno favorece o desenvolvimento sensorial e cognitivo, e protege a criança contra doenças crônicas e infecciosas, especialmente infecções respiratórias e diarréia ${ }^{1}$. Práticas adequadas de amamentação podem prevenir $57 \%$ das internações por infecções respiratórias e $72 \%$ por diarréia ${ }^{2}$.

Evidências científicas referem que a amamentação protege contra otite média em crianças até os dois anos de idade ${ }^{22}$, rinite alérgica em crianças menores de 5 anos $^{23}$ e promove a diminuição do risco de mal oclusões dentárias ${ }^{24}$. Estudo de revisão demonstrou que crianças amamentadas após os 12 meses de idade foram associadas com um risco aumentado de cáries dentárias, no entanto, não há associação entre a amamentação até 12 meses de idade com um risco aumentado de cáries ${ }^{25}$.

Em relação aos efeitos de longo prazo em crianças, evidências demonstram que a amamentação foi associada com uma redução de $13 \%$ do excesso de peso ou obesidade e pode reduzir as chances de diabetes tipo $2^{26}$. De acordo com Horta et al. ${ }^{27}$ a duração do aleitamento materno está associado com melhor desempenho em testes de inteligência. Em um estudo de coorte prospectivo realizado no Brasil, incluindo 30 anos de seguimento, demonstrou que os resultados ajustados de escores de inteligência foram significati- 
vamente maiores nos exclusivamente amamentados durante 12 meses ou mais ${ }^{28}$.

Além disso, a amamentação é benéfica para a saúde materna. Há fortes evidências de que o aleitamento por períodos maiores resulta em reduções significativas no risco de desenvolver câncer de mama ${ }^{29}$, além de promover o aumento do intervalo dos nascimentos e proteger contra o câncer de ovário e diabetes tipo $2^{2}$.

Para Chowdhury et al. ${ }^{30}$, o aleitamento materno exclusivo e predominante durante os seis meses após o parto foram evidenciados com o aumento da duração da amenorréia lactacional.

\section{A amamentação e a utilização de fármacos}

A transferência de fármacos para o leite materno pode ser maior durante os primeiros dias de lactação, denominada fase colostral ${ }^{31}$, pois existem grandes espaços entre as células alveolares, permitindo maior acesso à maioria dos fár$\operatorname{macos}^{32}$. Porém, devido ao volume limitado de colostro, doses mínimas são transferidas para o lactente durante esta fase ${ }^{31}$. Logo após a primeira semana, as células alveolares crescem, e consequentemente os espaços intracelulares são fechados, limitando assim a transferência dos fármacos no leite materno ${ }^{32}$.

Para que o profissional de saúde oriente à nutriz quanto à utilização de medicamentos, fatores relacionados com o fármaco, o lactente e a mãe devem ser considerados na avaliação dos riscos e benefícios do fármaco durante o aleitamento materno ${ }^{5}$.

A concentração do fármaco no plasma materno é um dos fatores mais importantes que deve ser considerado na exposição do lactente ao medicamento ${ }^{33}$.

No Quadro 1 estão representadas as características farmacocinéticas que influenciam na transferência de um fármaco para o leite materno.

Aspectos importantes como altos níveis do fármaco no leite materno, alta toxicidade, saúde infantil, tratamento medicamentoso a longo prazo e a idade da criança, devem ser considerados em relação ao aumento do risco de efeitos adversos em lactentes ${ }^{34}$.

Para avaliar o risco de eventos adversos na lactação após a ingestão materna de medicamentos, lactentes podem ser classificados como alto risco (recém-nascidos, prematuros ou com condições médicas agudas ou crônicas, como insuficiência renal), risco moderado (2 semanas- 6 meses) ou baixo risco (6-18 meses $)^{31}$.
Durante o período neonatal o sistema metabólico é imaturo, ocasionando meia-vida mais longa dos fármacos, o que pode justificar a maior frequência de reações adversas a medicamentos em recém-nascidos ${ }^{35}$. Além disso, lactentes recebem a dose mais elevada de um fármaco no leite materno em relação ao seu peso corporal no primeiro mês de vida ${ }^{36}$.

Uma revisão sobre reações adversas de medicamentos em lactentes constatou que 79\% das reações ocorreram nos 2 primeiros meses de vida ${ }^{37}$. Soussan et al. ${ }^{35}$ realizaram um estudo descritivo utilizando informações do banco de dados do Sistema de Farmacovigilância Francesa durante um período de 25,5 anos. Os resultados apontam que foram notificadas 276 reações adversas em 174 crianças amamentadas, sendo que as reações adversas mais frequentes foram neurológica $(28,6 \%)$ e gastrointestinal $(20,3 \%)$, e pelo menos dois terços dos casos notificados foram em recém-nascidos.

A duração do tratamento medicamentoso (curto ou a longo prazo), o tempo de exposição ao fármaco, o risco da mãe não receber o medicamento, a gravidade da condição a ser tratada são considerações importantes para serem avaliadas durante a amamentação ${ }^{5}$.

O volume de leite e o teor de gordura também devem ser avaliados. A concentração do fármaco no leite materno encontra-se mais elevada no início da manhã, pois o volume de leite é maior nesse período, entretanto o teor de gordura é maior no final da manhã ${ }^{21}$. Para diminuir a exposição do lactente ao fármaco, as nutrizes são orientadas a amamentar primeiro e após fazer uso do medicamento na menor dose terapêutica possível e com intervalos mais espaçados, assim o pico sérico do fármaco ocorrerá em período em que o lactente não está sendo amamentado ${ }^{38}$. Porém, este princípio não deve ser aplicado para medicamentos com meia-vida longa, como o diazepam. Portanto, deve ser realizada uma avaliação criteriosa em relação à real necessidade da utilização do medicamento em nutrizes, para evitar os potenciais efeitos tóxicos dos fármacos para o lactente ${ }^{39}$.

Para o fármaco atingir uma determinada concentração no plasma, ele deve ser absorvido sistemicamente na nutriz. Medicamentos administrados via oral ou injetáveis são frequentemente encontrados no leite. Já fármacos que são inalados ou administrados topicamente, como pomadas, cremes, colírios, sprays nasais, medicamentos inalatórios, não atingem níveis plasmáticos significativos, não sendo detectados em 
Quadro 1. Fatores que determinam a passagem de fármacos para o leite materno.

\begin{tabular}{|c|c|}
\hline Parâmetros farmacocinéticos & Referência \\
\hline $\begin{array}{l}\text { Gradiente de concentração: A passagem da maioria dos fármacos para o leite materno } \\
\text { ocorre através de difusão passiva seguindo um gradiente de concentração, ou seja, altos } \\
\text { níveis séricos consequentemente tendem a produzir altos níveis do fármaco no leite. }\end{array}$ & $\begin{array}{l}\text { Hotham e Hotham } \\
2015^{39} \\
\text { Nordeng et al., } 2012^{34} \\
\text { Berlin e Briggs } 2005^{40}\end{array}$ \\
\hline $\begin{array}{l}\text { Peso molecular: Fármacos com baixo peso molecular atravessam facilmente o leite } \\
\text { através dos pequenos poros nas paredes das células do epitélio mamário. Fármacos } \\
\text { com peso molecular elevado (maior que } 800 \text { daltons) como a insulina, a heparina e o } \\
\text { interferon, dificilmente passam para o leite materno. }\end{array}$ & $\begin{array}{l}\text { Nice e Luo } 2012^{21} \\
\text { Hotham e Hotham } \\
2015^{39}\end{array}$ \\
\hline $\begin{array}{l}\text { Volume de distribuição: Parâmetro utilizado para quantificar a distribuição de um } \\
\text { medicamento no organismo. Fármacos que possuem elevado volume de distribuição, } \\
\text { como por exemplo a sertralina, podem ingressar em diferentes compartimentos do } \\
\text { corpo e resultar em uma menor concentração plasmática materna, e consequentemente } \\
\text { em uma menor concentração no leite. Dessa forma, medicamentos que possuem um } \\
\text { volume de distribuição entre } 1 \text { e } 20 \mathrm{~L} / \mathrm{kg} \text { são compatíveis com a amamentação. }\end{array}$ & $\begin{array}{l}\text { Nice e Luo } 2012^{21} \\
\text { Hotham e Hotham } \\
2015^{39}\end{array}$ \\
\hline $\begin{array}{l}\text { Grau de ionização: As características ácido/ base de um fármaco são essenciais. O leite } \\
\text { materno é mais ácido }(\mathrm{pH} 7,1) \text { do que o plasma }(\mathrm{pH} 7,4) \text {. Fármacos que são bases } \\
\text { fracas, como por exemplo o propranolol (beta-bloqueador), permanecem na forma } \\
\text { ionizada no leite materno, favorecendo sua maior concentração. }\end{array}$ & $\begin{array}{l}\text { Berlin e Briggs } 2005^{40} \\
\text { Nice e Luo } 2012^{21}\end{array}$ \\
\hline $\begin{array}{l}\text { Ligação à proteínas: Quanto maior a ligação à proteínas do plasma materno, menor } \\
\text { quantidade de fármaco transfere-se para o leite, reduzindo à exposição do lactente } \\
\text { ao medicamento. Um fármaco com ligação maior que } 80 \% \text { às proteínas plasmáticas } \\
\text { é considerado ideal para ser utilizado durante a amamentação. O ibuprofeno é um } \\
\text { fármaco com uma capacidade elevada de ligação às proteínas }(99 \%) \text {. }\end{array}$ & $\begin{array}{l}\text { Davanzo et al., } 2013^{41} \\
\text { Nice e Luo } 2012^{21} \\
\text { Rainsford } 2009^{42}\end{array}$ \\
\hline $\begin{array}{l}\text { Biodisponibilidade oral: É um dos principais parâmetros farmacocinéticos. Descreve } \\
\text { a fração de uma dose de uma droga administrada por via oral que atinge a circulação } \\
\text { sistêmica. Um fármaco com baixa biodisponibilidade oral é compatível com a } \\
\text { amamentação e o risco de efeitos adversos pode ser menor, pois a absorção pela } \\
\text { circulação sistêmica do lactente através do intestino é baixa. }\end{array}$ & $\begin{array}{l}\text { Davanzo et al., } 2013^{41} \\
\text { Bertino et al., } 2012^{43}\end{array}$ \\
\hline $\begin{array}{l}\text { Meia-vida: Fármacos com menores meias-vidas diminuem a exposição do lactente } \\
\text { às drogas no leite materno. Se o tempo de meia-vida é maior que } 12 \text { a } 24 \text { horas, o } \\
\text { fármaco tende a permanecer no plasma materno por mais tempo, e por conseguinte, } \\
\text { poderá acumular-se no leite materno. Somente após } 5 \text { meias-vidas, quase todo } \\
\text { fármaco }(97 \%) \text { é excretado. }\end{array}$ & $\begin{array}{l}\text { Nice e Luo } 2012^{21} \\
\text { Davanzo et al., } 2013^{41}\end{array}$ \\
\hline $\begin{array}{l}\text { Lipossolubilidade: Fármacos que são lipossolúveis tendem a penetrar no leite em } \\
\text { concentrações mais elevadas, em especial os que são ativos no sistema nervoso central. }\end{array}$ & $\begin{array}{l}\text { Hale } 2016^{32} \\
\text { Burkey e Holmes } \\
2013^{33}\end{array}$ \\
\hline
\end{tabular}

quaisquer concentrações significativas no leite materno ${ }^{34}$.

\section{Métodos de estimativa de exposição ao fármaco}

A quantificação da exposição do lactente aos fármacos via leite materno torna-se uma ferramenta útil na prática clínica para a determinação da segurança do uso de fármacos durante a lactação9.

Um dos parâmetros utilizados para estimar a transferência do fármaco no leite materno segundo Davanzo et al. ${ }^{41}$ é a razão leite/plasma. Está relacionada entre a concentração do fárma- co no leite materno dividido pela sua concentração no plasma materno:

$$
\text { Razão leite/plasma: } \frac{\text { Concentração do fármaco no leite }}{\text { Concentração do fármaco no plasma }}
$$

Essa razão pode ser estimada a partir das medições do fármaco no leite e no plasma após uma única dose do medicamento administrada ou da área sob a curva calculada das medições do fármaco no leite e no plasma em um intervalo de tempo ${ }^{44}$.

Se a razão leite/plasma for superior a 1,0 demonstra que o fármaco pode estar em concentrações elevadas no leite materno ${ }^{41}$, porém essa informação pode ser clinicamente irrelevante se 
a quantidade de fármaco que o lactente ingeriu por unidade de tempo for muito mais baixa do que a necessária para fins terapêuticos, assim o nível de exposição pode ser considerado baixo, independentemente da razão leite/plasma ${ }^{45}$.

Outros parâmetros utilizados são a dose teórica e a dose relativa do lactente. A dose teórica do lactente pode ser calculada multiplicando-se a concentração do fármaco no leite materno com o volume de leite que o lactente ingere (fórmula $1)^{34}$. O volume de leite ingerido é variável, pois depende da idade da criança e se a alimentação é exclusiva com leite materno ${ }^{31}$. Estima-se que o lactente amamentado exclusivamente com leite materno consuma $150 \mathrm{~mL} / \mathrm{kg} / \mathrm{dia}^{39}$.

Fórmula 1: Dose teórica do lactente $(\mathrm{em} \mathrm{mg} / \mathrm{dia})=$ concentração do fármaco no leite materno $(\mathrm{em} \mathrm{mg} / \mathrm{L}) \times 0,15 \mathrm{~L} / \mathrm{kg} /$ dia $\times$ peso corporal da criança $(\mathrm{em} \mathrm{Kg})$

A concentração do fármaco no leite pode ser calculada pela concentração máxima (Cmáx) observada de fármaco no leite durante um intervalo de tempo ou pela concentração média do fármaco no leite (Cav) medida ao longo de um intervalo de dose a partir da fórmula $\mathrm{AUC}_{0-\tau} / \tau$, onde $\tau=$ duração do intervalo de dose em horas ${ }^{44}$.

A dose relativa do lactente (do inglês Relative Infant Dose- RID) fornece um método padronizado que estima a porcentagem da quantidade de dose materna recebida pelo lactente. Pode ser calculada através da fórmula $2^{21,31,34}$ :

Fórmula 2: Dose relativa do lactente $(\%)=$ dose do lactente $(\mathrm{mg} /$ $\mathrm{kg} / \mathrm{dia}) /$ dose materna $(\mathrm{mg} / \mathrm{kg} / \mathrm{dia}) \times 100$

A transferência do fármaco para o leite materno é considerada mínima quando $\mathrm{RID}<2 \%$ e alta quando RID $>10 \%{ }^{34}$. Quando a dose relativa do lactente é inferior à $10 \%$ da dose materna o fármaco é considerado seguro durante a amamentação $0^{21}$. Dessa forma, torna-se fundamental que cada situação seja avaliada de acordo com a toxicidade do medicamento ${ }^{31}$, e com as características individuais da nutriz e do lactente.

\section{Classificação dos fármacos para uso durante a lactação}

Segundo Hale e Rowe ${ }^{7}$ os medicamentos são classificados em categorias de risco na lactação:

- Nível L1: Fármacos seguros ao mais alto nível. Foram administrados em um número grande de nutrizes sem qualquer aumento de efeitos adversos observados no lactente.
- Nível L2: Fármacos considerados seguros. Foram estudados em número limitado de nutrizes sem aumento de efeitos adversos no lactente.

- Nivel L3: Fármacos moderadamente seguros. Não há estudos controlados em nutrizes e o risco de efeitos adversos no lactente é possível. Estes fármacos devem ser administrados somente se o benefício justificar o risco potencial para o lactente.

- Nivel L4: Fármacos possivelmente perigosos. Há evidência positiva de risco para o lactente ou na produção de leite materno. No entanto, os benefícios do uso destes fármacos podem ser admissíveis apesar do risco para o lactente, em situações em que o fármaco é necessário como em casos de risco de vida ou doença grave.

- Nível L5: Fármacos que possuem um elevado risco de causar danos significativos no lactente. Uso contraindicado em nutrizes.

$\mathrm{Na}$ Tabela 1 estão apresentadas as classes farmacológicas que podem ser comumente utilizadas durante a amamentação e a segurança do uso desses fármacos.

\section{Anti-hipertensivos}

A hipertensão é uma das doenças de preocupação de saúde pública mundial e que apresenta alta e crescente prevalência ${ }^{46}$. Na literatura, as classes terapêuticas mais comuns de fármacos anti-hipertensivos são: beta-bloqueadores, bloqueadores dos canais de cálcio, inibidores da enzima conversora de angiotensina (IECA) e diuréticos, conforme Tabela 2. A maioria dos fármacos pertencentes a estas classes são apropriados para o uso durante a amamentação. Porém, quando se utiliza os beta-bloqueadores recomenda-se a monitorização do lactente quanto aos efeitos de bradicardia, hipotensão e letargia ${ }^{31}$.

\section{Corticosteroides}

A maioria dos corticosteroides é excretada no leite materno em pequenas quantidades ${ }^{7}$. Em um grupo de seis nutrizes que receberam tratamento com prednisolona em doses de 10 a $80 \mathrm{mg} / \mathrm{dia}$, as concentrações no leite foram de $5 \%$ a $25 \%$ dos níveis séricos maternos. A exposição do lactente ao fármaco foi mínima e o aleitamento materno foi considerado seguro em doses de $20 \mathrm{mg}$ de prednisolona, administradas uma ou duas vezes ao dia. Em doses mais elevadas, a exposição pode ser minimizada evitando-se a amamentação durante as primeiras 4 horas após a administração do fár$\mathrm{maco}^{47}$. Dessa forma, em doses pequenas, a maio- 
Tabela 1. Recomendações sobre o uso de fármacos durante a amamentação.

\begin{tabular}{|c|c|c|c|c|c|c|}
\hline \multicolumn{7}{|c|}{ Avaliação do risco na lactação } \\
\hline \multirow[t]{2}{*}{$\begin{array}{l}\text { Classe } \\
\text { farmacológica/ } \\
\text { Medicamento }\end{array}$} & RID (\%) & $\begin{array}{c}\text { Grau } \\
\text { de risco } \\
\text { do uso }^{7}\end{array}$ & & Estudos & $\begin{array}{l}\text { Lactmed } 2016^{8} \text { (incluindo reações } \\
\text { adversas no lactente) }\end{array}$ & Recomendação \\
\hline & & & $\mathbf{N}$ & Autor/ Ano & & \\
\hline \multicolumn{7}{|l|}{ Antibióticos } \\
\hline $\begin{array}{l}\text { Amoxicilina+ } \\
\text { Clavulanato }\end{array}$ & 0.9 & $\mathrm{~L} 1$ & 67 & $\begin{array}{l}\text { Benyamini } \\
\text { et al., } 2005^{48}\end{array}$ & $\begin{array}{l}\text { Monitorar o lactente para reações como agitação, } \\
\text { diarréia e erupções cutâneas que ocorrem } \\
\text { eventualmente. }\end{array}$ & Seguro \\
\hline Azitromicina & 5.9 & $\mathrm{~L} 2$ & 20 & $\begin{array}{l}\text { Salman et } \\
\text { al., } 2016^{49}\end{array}$ & $\begin{array}{l}\text { Monitorar o lactente quanto à possíveis efeitos } \\
\text { como alterações na flora gastrointestinal (diarréia) } \\
\text { e candidíase. }\end{array}$ & Seguro \\
\hline Ciprofloxacino & $2.1-6.34$ & L3 & 10 & $\begin{array}{l}\text { Giamarellou } \\
\text { et al., } 1989^{50}\end{array}$ & $\begin{array}{l}\text { Possíveis efeitos no lactente sobre a flora } \\
\text { gastrointestinal (diarréia) e candidíase. Deve-se } \\
\text { evitar a amamentação durante } 3 \text { a } 4 \text { horas após a } \\
\text { dose de ciprofloxacino para diminuir a exposição } \\
\text { do lactente ao fármaco no leite materno. }\end{array}$ & $\begin{array}{l}\text { Moderadamente } \\
\text { seguro }\end{array}$ \\
\hline Cefalexina & $0.5-1.5$ & $\mathrm{~L} 1$ & 6 & $\begin{array}{l}\text { Kafetzis et } \\
\text { al., } 1981^{51}\end{array}$ & $\begin{array}{l}\text { Foram relatados com a administração de } \\
\text { cefalexina alterações na flora gastrointestinal, } \\
\text { resultando em diarréia ou aftas no lactente. }\end{array}$ & Seguro \\
\hline \multicolumn{7}{|c|}{ Antinflamatórios não- esteróides } \\
\hline Diclofenaco & ND & $\mathrm{L} 2$ & 30 & $\begin{array}{l}\text { Hirose et al., } \\
1996^{52}\end{array}$ & $\begin{array}{l}\text { Não há relatos de reações adversas nos lactentes, } \\
\text { apenas um lactente desenvolveu urticária no } 15^{\circ} \\
\text { dia de vida, porém a criança tinha recebido } 7 \text { dias } \\
\text { antes a vacinação contra hepatite } \mathrm{B} \text {, o que pode ter } \\
\text { causado a reação. }\end{array}$ & Seguro \\
\hline Naproxeno & 3.3 & L3 & 60 & $\begin{array}{l}\text { Sammour et } \\
\text { al., } 2011^{53}\end{array}$ & $\begin{array}{l}\text { Efeitos adversos em crianças amamentadas } \\
\text { são incomuns. Devido à meia-vida longa do } \\
\text { naproxeno, foi relatada reação adversa grave } \\
\text { em recém-nascido. Sangramento prolongado, } \\
\text { trombocitopenia e anemia aguda foi relatado em } \\
\text { lactente aos } 7 \text { dias de idade. Dois casos relatados } \\
\text { de sonolência e um caso de vômito em lactentes. }\end{array}$ & $\begin{array}{l}\text { Moderadamente } \\
\text { seguro }\end{array}$ \\
\hline Ibuprofeno & $0.1-0.7$ & L1 & 13 & $\begin{array}{l}\text { Rigourd et } \\
\text { al., } 2014^{54}\end{array}$ & $\begin{array}{l}\text { Não foram reportados efeitos adversos em } \\
\text { lactentes, devido aos níveis extremamente baixos } \\
\text { de ibuprofeno no leite materno. }\end{array}$ & Seguro \\
\hline Celecoxibe & $0.3-0.7$ & $\mathrm{~L} 2$ & 6 & $\begin{array}{l}\text { Gardiner et } \\
\text { al., } 2006^{55}\end{array}$ & $\begin{array}{l}\text { Não foram reportados efeitos adversos em } \\
\text { lactentes, devido aos baixos níveis de celecoxibe no } \\
\text { leite materno. }\end{array}$ & Seguro \\
\hline \multicolumn{7}{|l|}{ Analgésicos } \\
\hline $\begin{array}{l}\text { Ácido } \\
\text { acetilsalicílico } \\
\text { (AAS) }\end{array}$ & $2.5-10.8$ & L2 & 6 & $\begin{array}{l}\text { Jamali e } \\
\text { Keshavarz } \\
1981^{56}\end{array}$ & $\begin{array}{l}\text { Foram reportados efeitos adversos, tais como } \\
\text { acidose metabólica em um lactente aos } 16 \text { dias } \\
\text { de idade; trombocitopenia, febre, anorexia e } \\
\text { petéquias em lactente aos } 5 \text { meses de idade. }\end{array}$ & Seguro \\
\hline Dipirona & $1.2-3$ & L4 & 8 & $\begin{array}{l}\text { Zylber-Katz } \\
\text { et al., } 1986^{57}\end{array}$ & $\begin{array}{l}\text { Pode causar reações adversas, tais como a } \\
\text { agranulocitose e foi reportado um caso de } \\
\text { episódios de cianose em lactente aos } 42 \text { dias } \\
\text { de idade. Após a administração de uma dose } \\
\text { de dipirona, o fármaco e seus metabólitos são } \\
\text { eliminados do leite materno durante } 48 \text { horas. }\end{array}$ & $\begin{array}{l}\text { Possivelmente } \\
\text { perigoso }\end{array}$ \\
\hline Paracetamol & $\begin{array}{r}8.8^{-} \\
24.2^{*}\end{array}$ & $\mathrm{~L} 1$ & 4 & $\begin{array}{l}\text { Notarianni } \\
\text { et al., } 1987^{58}\end{array}$ & $\begin{array}{l}\text { Efeitos adversos em recém-nascidos são raros. Foi } \\
\text { reportado uma erupção cutânea maculopapular } \\
\text { no tronco superior e na face em um lactente aos } 2 \\
\text { meses de idade. }\end{array}$ & Seguro \\
\hline Tramadol & 2.86 & L3 & 75 & $\begin{array}{l}\text { Ilett et al., } \\
2008^{59}\end{array}$ & $\begin{array}{l}\text { Monitorar o lactente para aumento da sonolência, } \\
\text { dificuldades respiratórias ou fraqueza e dificuldade } \\
\text { de amamentação. }\end{array}$ & $\begin{array}{l}\text { Moderadamente } \\
\text { seguro }\end{array}$ \\
\hline
\end{tabular}


Tabela 1. Recomendações sobre o uso de fármacos durante a amamentação.

\begin{tabular}{|c|c|c|c|c|c|c|}
\hline \multicolumn{7}{|c|}{ Avaliação do risco na lactação } \\
\hline $\begin{array}{c}\text { Classe } \\
\text { farmacológica/ } \\
\text { Medicamento } \\
\end{array}$ & RID (\%) & $\begin{array}{c}\text { Grau } \\
\text { de risco } \\
\text { do uso }^{7}\end{array}$ & & Estudos & $\begin{array}{l}\text { Lactmed } 2016^{8} \text { (incluindo reações } \\
\text { adversas no lactente) }\end{array}$ & Recomendação \\
\hline & & & $\mathbf{N}$ & Autor/Ano & & \\
\hline Codeína & $0.6-8.1$ & L4 & 238 & $\begin{array}{l}\text { Kelly et al., } \\
2013^{60}\end{array}$ & $\begin{array}{l}\text { Pode causar no lactente aumento da sonolência, } \\
\text { dificuldades respiratórias ou fraqueza, dificuldade } \\
\text { de amamentação, depressão do sistema nervoso } \\
\text { central e até mesmo a morte. }\end{array}$ & $\begin{array}{l}\text { Possivelmente } \\
\text { perigoso }\end{array}$ \\
\hline \multicolumn{7}{|l|}{ Antidepressivos } \\
\hline Fluoxetina & 1.6- 14.6 & L2 & 11 & $\begin{array}{l}\text { Epperson et } \\
\text { al., } 2003^{61}\end{array}$ & $\begin{array}{l}\text { Monitorar o lactente para efeitos adversos, tais } \\
\text { como cólicas, pieguice, sedação e ganho de peso } \\
\text { adequado. }\end{array}$ & Seguro \\
\hline Paroxetina & $1.2-2.8$ & L2 & 27 & $\begin{array}{l}\text { Merlob et } \\
\text { al., } 2004^{62}\end{array}$ & $\begin{array}{l}\text { Efeitos adversos foram relatados em um lactente, } \\
\text { tais como agitação e dificuldade na alimentação. } \\
\text { Em nutrizes em uso de paroxetina durante o } \\
\text { terceiro trimestre de gravidez e que amamentaram } \\
\text { seus filhos, relataram efeitos adversos incluindo } \\
\text { estado de alerta, constipação, sonolência e } \\
\text { irritabilidade. Monitorar o lactente em relação ao } \\
\text { ganho de peso adequado. }\end{array}$ & Seguro \\
\hline Sertralina & $0.4-2.2$ & L1 & 26 & $\begin{array}{l}\text { Stowe et al., } \\
2003^{63}\end{array}$ & $\begin{array}{l}\text { Mioclonia neonatal benigna do sono ocorreu } \\
\text { em um lactente de } 4 \text { meses de idade e agitação } \\
\text { foi reportado em um lactente. Hipertermia, } \\
\text { distúrbios da regulação do tônus muscular e } \\
\text { choro estridente foram relatados em lactente } \\
\text { prematuro. Monitorar o recém-nascido em relação } \\
\text { à sedação, ganho de peso adequado e dificuldades } \\
\text { de alimentação. }\end{array}$ & Seguro \\
\hline Escitalopram & $5.2-7.9$ & L2 & 8 & $\begin{array}{l}\text { Rampono } \\
\text { et al., } 2006^{64}\end{array}$ & $\begin{array}{l}\text { Monitorar o lactente para sonolência, } \\
\text { especialmente nos mais jovens, quando se utiliza } \\
\text { combinações de fármacos psicotrópicos e lactentes } \\
\text { alimentados exclusivamente com leite materno. } \\
\text { Foram relatados efeitos adversos tais como } \\
\text { irritabilidade, vômito e febre em lactente aos } 4 \\
\text { meses de idade. }\end{array}$ & Seguro \\
\hline Amitriptilina & $1.9-2.8$ & L2 & 1 & $\begin{array}{l}\text { Brixen- } \\
\text { Rasmussen, } \\
\text { et al., } 1982^{65}\end{array}$ & $\begin{array}{l}\text { Não foram relatados efeitos adversos imediatos, } \\
\text { pois os níveis de amitriptilina e seus metabólitos } \\
\text { no leite materno são baixos. }\end{array}$ & Seguro \\
\hline \multicolumn{7}{|l|}{ Antifúngicos } \\
\hline Cetoconazol & 0.3 & L2 & 1 & $\begin{array}{l}\text { Moretti et } \\
\text { al., } 1995^{66}\end{array}$ & $\begin{array}{l}\text { Para diminuir a exposição do lactente ao } \\
\text { cetoconazol, recomenda-se evitar a amamentação } \\
\text { a partir de } 2 \text { a } 5 \text { horas após a administração da } \\
\text { dose. Nenhum efeito adverso foi relatado. }\end{array}$ & Seguro \\
\hline Fluconazol & $\begin{array}{l}16.4- \\
21.5^{+}\end{array}$ & L2 & 96 & $\begin{array}{l}\text { Moorhead } \\
\text { et al., } 2011^{67}\end{array}$ & $\begin{array}{l}\text { Foram reportados efeitos adversos possivelmente } \\
\text { causados por fluconazol em lactentes, tais como } \\
\text { bochechas coradas, desconforto gastrointestinal e } \\
\text { fezes mucosas. }\end{array}$ & Seguro \\
\hline \multicolumn{7}{|l|}{ Ansiolíticos } \\
\hline Alprazolam & 8.5 & L3 & 8 & $\begin{array}{l}\text { Oo et al., } \\
1995^{68}\end{array}$ & $\begin{array}{l}\text { Foram reportados efeitos adversos em recém- } \\
\text { nascidos, incluindo aumento da irritabilidade, } \\
\text { sonolência e sedação. }\end{array}$ & $\begin{array}{l}\text { Moderadamente } \\
\text { seguro }\end{array}$ \\
\hline Diazepam & $0.88-7.14$ & L3 & 1 & $\begin{array}{l}\text { Dusci et al., } \\
1990^{69}\end{array}$ & $\begin{array}{l}\text { Efeito sedativo e sonolência foram reportados em } \\
\text { lactentes. Perda de peso, letargia e efeito sedativo } \\
\text { ocorreu em lactente com } 8 \text { dias de idade. }\end{array}$ & $\begin{array}{l}\text { Moderadamente } \\
\text { seguro }\end{array}$ \\
\hline
\end{tabular}


Tabela 1. Recomendações sobre o uso de fármacos durante a amamentação.

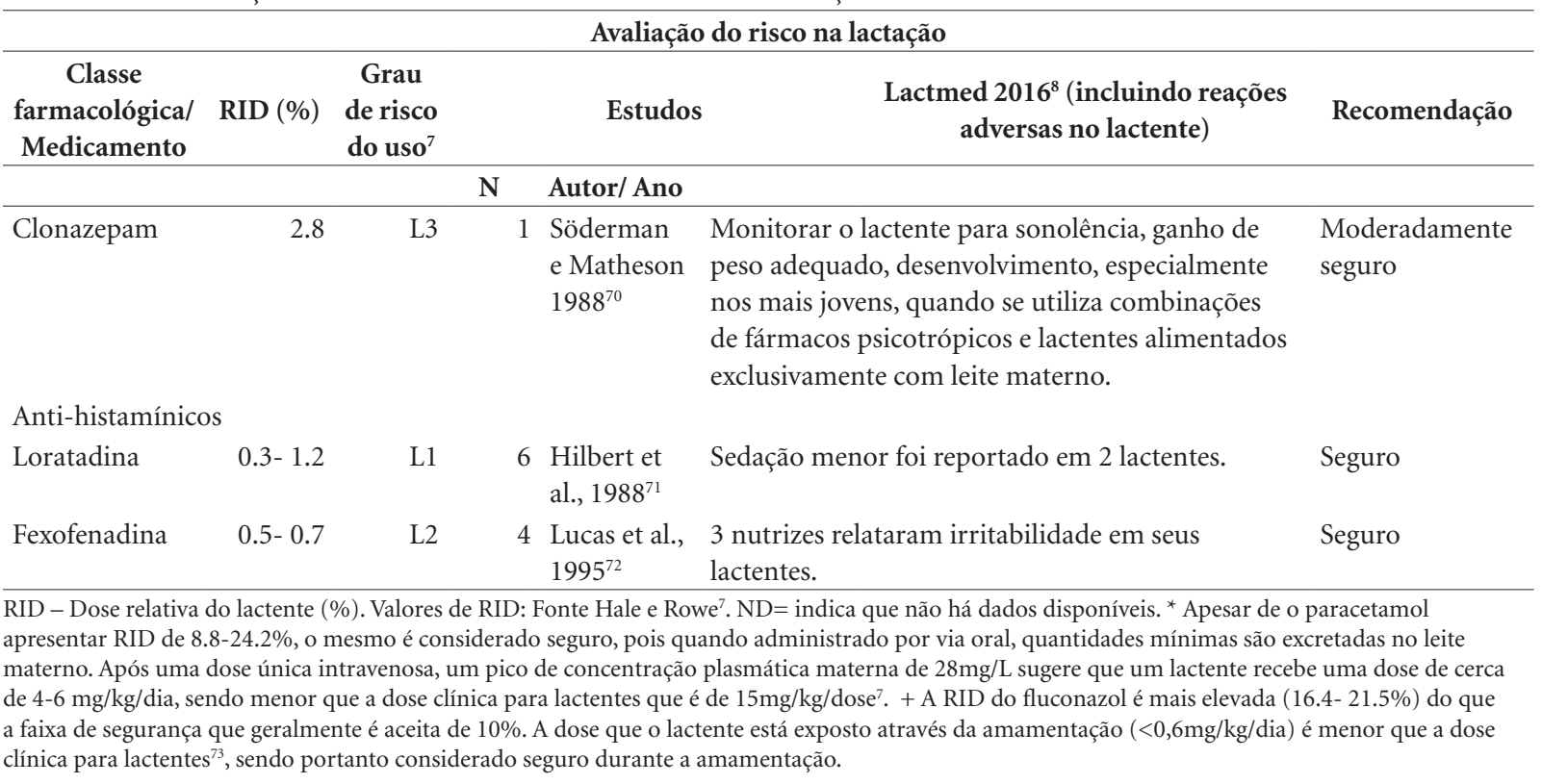

Tabela 2. Avaliação da segurança do uso de fármacos anti-hipertensivos durante a amamentação.

\begin{tabular}{|c|c|c|c|c|c|c|}
\hline \multicolumn{2}{|c|}{ Anti-hipertensivos } & \multirow{2}{*}{$\begin{array}{c}\begin{array}{c}\text { Grau de } \\
\text { risco do } \\
\text { uso }^{7}\end{array} \\
\text { L2 }\end{array}$} & \multirow{2}{*}{$\begin{array}{r}\begin{array}{c}\text { Tempo } \\
\text { meia-vida } \\
\text { (horas) }\end{array} \\
6-7\end{array}$} & \multirow{2}{*}{$\begin{array}{c}\begin{array}{c}\text { Razão } \\
\text { leite/ } \\
\text { plasma }\end{array} \\
3-3.72\end{array}$} & \multirow{2}{*}{$\begin{array}{l}\begin{array}{l}\text { RID } \\
(\%)\end{array} \\
1.4\end{array}$} & \multirow[t]{2}{*}{ Recomendação } \\
\hline Beta-bloqueadores & Metoprolol & & & & & \\
\hline \multirow{6}{*}{$\begin{array}{l}\text { Bloqueadores dos } \\
\text { canais de cálcio }\end{array}$} & Propranolol & $\mathrm{L} 2$ & $3-5$ & 0.5 & $0.3-0.5$ & Seguro \\
\hline & Atenolol & L3 & $6-7$ & $1.5-6.8$ & 6.6 & Moderadamente seguro \\
\hline & Amlodipino & L3 & $30-50$ & ND & $1.72-3.15$ & Moderadamente seguro \\
\hline & Nifedipino & $\mathrm{L} 2$ & $1.8-7$ & 1.0 & $2.3-3.4$ & Seguro \\
\hline & Verapamil & $\mathrm{L} 2$ & $3-7$ & 0.94 & 0.2 & Seguro \\
\hline & Diltiazem & L3 & $3.5-6$ & 1.0 & 0.9 & Moderadamente seguro \\
\hline \multirow{2}{*}{$\begin{array}{l}\text { Inibidores da enzima } \\
\text { conversora de } \\
\text { angiotensina (IECA) }\end{array}$} & Captopril & L2 & 2.2 & 0.012 & 0.02 & Seguro \\
\hline & Enalapril & $\mathrm{L} 2$ & $\begin{array}{r}35 \\
\text { (metabólito) }\end{array}$ & ND & $0.07-0.2$ & Seguro \\
\hline \multirow[t]{2}{*}{ Diuréticos } & Hidroclorotiazida & $\mathrm{L} 2$ & $5.6-14.8$ & 0.25 & ND & Seguro \\
\hline & Furosemida & L3 & 1.5 & ND & ND & Moderadamente seguro \\
\hline
\end{tabular}

RID - Dose relativa do lactente (\%). ND= indica que não há dados disponíveis.

Adaptado de Hale e Rowe ${ }^{7}$.

ria dos corticosteroides não são contraindicados durante o período de amamentação?.

Os corticosteroides tópicos são provavelmente seguros durante a lactação e a exposição do recém-nascido através do leite materno é míni$\mathrm{ma}^{74}$. No entanto, corticosteroides tópicos de alta potência, tais como dipropionato ou valerato de betametasona nas apresentações de $0,05 \%$ creme ou pomada e $0,1 \%$ pomada, triancinolona na apresentação de $0,5 \%$ creme ou pomada, não devem ser usados nos mamilos e recomenda-se cautela se usados em grandes áreas do corpo ${ }^{7}$.

\section{Antidiabéticos}

A metformina é um fármaco de primeira linha para o tratamento de diabetes mellitus tipo $2^{75}$. Este medicamento pertence à classe das bi- 
guanidas e apresenta biodisponibilidade oral de $50 \%{ }^{7}$. No estudo de Briggs et al. ${ }^{76}$ realizado com 5 nutrizes e 3 lactentes, o pico médio e as concentrações mínimas da metformina no leite materno foram de $0,42 \mu \mathrm{g} / \mathrm{mL}$ e $0,39 \mu \mathrm{g} / \mathrm{mL}$, respectivamente, e a dose relativa do lactente foi de $0.65 \%$. As concentrações de glicose no sangue foram monitoradas nos 3 lactentes no período de 4 horas após a amamentação e apresentaram-se dentro do limite normal. Os autores concluíram que a metformina é excretada no leite materno, mas em quantidades clinicamente insignificantes, sendo considerada compatível com a amamentação.

A glibenclamida é uma sulfoniluréia de segunda geração utilizada para o tratamento farmacológico de diabetes mellitus tipo $2^{7}$. No estudo de Feig et al. ${ }^{77}$ realizado com 6 nutrizes em que foram administradas uma dose única de $5 \mathrm{mg}$ de glibenclamida e 2 nutrizes em que foram administradas uma dose única de $10 \mathrm{mg}$, em ambas as doses, o fármaco não foi detectado no leite materno (limite de detecção $0.005 \mu \mathrm{g} / \mathrm{mL}$ ). Em um grupo de 5 nutrizes foram administradas doses diárias de $5 \mathrm{mg}$ de glibenclamida ou glipizida. Ambos os fármacos não foram detectados no leite materno. Não foi observada hipoglicemia nos 3 lactentes em estudo. Glibenclamida e glipizida nas doses testadas foram consideradas compatíveis com a amamentação.

Nutrizes com diabetes em uso de insulina podem amamentar os seus bebês. A necessidade de administração de insulina é reduzida cerca de $60 \%$ da dose pré-gravidez, em virtude da falta de influência hormonal placentária após o parto ${ }^{78}$. A insulina é um componente normal do leite materno e há evidências de que exerce um papel importante em lactentes na prevenção primária de diabetes tipo $1^{79}$.

A insulina exógena é excretada no leite materno, incluindo as insulinas biossintéticas mais recentes, são elas: insulina lispro, aspártica, detemir ${ }^{8}$. No estudo de Whitmore et al. ${ }^{80}$ realizado com 5 nutrizes sem diabetes, 4 nutrizes com diabetes tipo 1 e 5 nutrizes com diabetes tipo 2, os achados demonstraram que a insulina é transportada para o leite materno, porém não houve diferença nos níveis de insulina no leite materno em nutrizes sem diabetes, com diabetes tipo 2 (não em terapia de insulina) e com diabetes tipo 1 (em terapia com insulina). Os autores sugerem que a insulina exógena utilizada para o tratamento de diabetes do tipo 1 é transportada para o leite com uma afinidade semelhante à insulina endógena em nutrizes sem diabetes.

\section{Antirretrovirais}

É controversa a indicação de aleitamento materno em mães infectadas pelo HIV (Human Immunodeficiency Virus- Vírus da Imunodeficiência Humana). A orientação da Organização Mundial da Saúde para as nutrizes é que elas somente devem cessar a amamentação quando uma dieta sem o leite materno for viável, acessível, aceitável, segura, sustentável e nutricionalmente adequada, caso contrário é recomendado o aleitamento materno exclusivo durante os primeiros 6 meses $^{81}$. Diretrizes do Ministério da Saúde do Brasil, atualizadas em 2014, recomendam que a criança nascida de mãe infectada pelo HIV não seja amamentada, e sugere a substituição do leite materno por fórmula infantil ${ }^{82}$. Entretanto, novas discussões têm surgido em relação à amamentação de crianças nascidas de mães infectadas pelo $\mathrm{HIV}^{83}$.

A administração de regimes triplos de medicamentos antirretrovirais em nutrizes infectadas pelo HIV, com o objetivo de diminuir o risco de transmissão ao recém-nascido, têm demonstrado ocasionar mínima toxicidade nas crianças amamentadas ${ }^{84}$. No estudo de Palombi et al. ${ }^{85}$ realizado com 66 mulheres, destas 29 receberam zidovudina (ZDV), lamivudina (3TC) e nevirapina (NVP), 28 receberam estavudina (d4T), lamivudina (3TC) e nevirapina (NVP), e 9 receberam zidovudina (ZDV), lamivudina (3TC) e lopinavir/ ritonavir (LPV/r). A razão leite/plasma foi de 0,6 para ZDV, 3TC e NPV, 1,0 para d4T e 0,4 para LPV/r. Somente nevirapina atingiu níveis significativos nos recém-nascidos. Não foi encontrada nenhuma correlação significativa entre as concentrações dos fármacos e eventos adversos como anemia infantil. Os autores consideraram seguro e eficaz para a nutriz e o recém-nascido a administração materna destes 3 regimes de até 6 meses após o parto.

No entanto, o uso prolongado de fármacos antirretrovirais durante a lactação pode estar associado ao aumento do risco de toxicidade hematológica no lactente ${ }^{86,87}$.

\section{Galactagogos}

Fármacos que estimulam a produção do leite materno são denominados de galactagogos ${ }^{39}$. Dessa forma, a produção de leite pode ser aumentada com a utilização de antagonistas da dopamina, tais como a domperidona e a metoclopramida, que estimulam a liberação dos níveis de prolactina ${ }^{31}$. 
Tendo em vista a preocupação sobre os potenciais riscos graves para a saúde associados com o uso de domperidona em nutrizes para aumentar a produção de leite materno, a mesma não é aprovada pelo Food and Drug Administration (FDA) nos EUA para qualquer indicação. Embora, a domperidona seja aprovada em vários países para o tratamento de certos distúrbios gástricos, com exceção dos EUA, este fármaco não é aprovado em qualquer país, incluindo os EUA, para aumentar a produção de leite materno em nutrizes. Maiores níveis de domperidona no sangue estão associados a riscos mais elevados de eventos adversos que incluem arritmias cardíacas, parada cardíaca e morte súbita ${ }^{88,89}$. No entanto, nos países em que a domperidona está disponível, é considerado um fármaco de escolha como galactagogo (uso off label) ${ }^{90}$.

A dose habitual de metoclopramida é de 10 a $15 \mathrm{mg}$ por via oral administrados três vezes ao $\mathrm{dia}^{7}$. A dose de metoclopramida presente no leite humano é baixa, de 6 a $24 \mu \mathrm{g} / \mathrm{kg} /$ dia para lactentes estudados no pós-parto precoce e 1 a $13 \mu \mathrm{g} / \mathrm{kg} / \mathrm{dia}$ para os lactentes estudados após 8 a 12 semanas pós-parto. Não foi relatado evento adverso quando as nutrizes receberam metoclopramida $10 \mathrm{mg}$ administrados 3 vezes ao dia durante 2 semanas ${ }^{91}$. Porém, deve-se monitorar a nutriz para os eventos adversos deste fármaco, que incluem depressão grave, sintomas extrapiramidais e diarreia ${ }^{7}$.

Outros fármacos como a sulpirida e a clorpromazina também são utilizados como galactagogos $^{90}$, porém há a possibilidade de desenvolver efeitos adversos maternos, incluindo reações extrapiramidais e sedação ${ }^{7}$.

\section{Fármacos que suprimem a lactação}

Existem ainda os fármacos que podem reduzir a produção de leite materno, tais como a bromocriptina, contraceptivos orais combinados a estrógenos/progestágenos, dienoestrol, diuréticos tiazídicos, estilbestrol, estradiol e piridoxina ${ }^{92}$.

A levodopa pode suprimir a produção de leite materno, não sendo recomendado o seu uso durante o período de lactação ${ }^{7}$. Estudo recente tem demonstrado o uso de cabergolina para inibir a lactação em certas condições médicas, tais como aborto e morte neonatal ${ }^{93}$.

\section{Fármacos contraindicados}

Atualmente, poucos fármacos têm uso comprovadamente proibidos durante o período da amamentação, conforme Quadro 2.

\section{Nove pontos-chave para o uso de fármacos durante a lactação}

1. Deve-se avaliar risco $\mathrm{x}$ benefício do tratamento farmacológico para a nutriz e o lactente e se há a necessidade de interromper a amamentação ${ }^{92}$;

2. Em geral, medicamentos considerados seguros possuem dose relativa do lactente inferior à $10 \%^{7}$;

3. Selecionar fármacos para os quais há dados baseados em evidências, e não aqueles que são introduzidos recentemente ${ }^{10}$;

4. Medicamentos administrados nos primeiros 3 a 4 dias após o parto, geralmente produzem níveis subclínicos no lactente, devido ao volume limitado de leite ${ }^{7}$;

5. Recomenda-se escolher fármacos com as seguintes características: meia-vida curta, forte ligação às proteínas, baixa biodisponibilidade oral, elevado peso molecular ${ }^{7}$ e elevado volume de distribuição ${ }^{21,39}$;

6. Quando possível utilizar tratamentos tópicos ou locais ${ }^{10}$;

7. Prestar atenção para quaisquer sintomas ou sinais incomuns no recém-nascido ${ }^{43}$;

8. Para minimizar a exposição do lactente ao fármaco, orientar a nutriz para realizar a amamentação antes da administração do medicamento $^{39}$;

9. Considerar o paracetamol e o ibuprofeno como melhores alternativas para o alívio da dor em nutrizes. Evitar a administração em doses elevadas de aspirina quando o lactente apresenta uma síndrome viral, pois neste caso a aspirina está associada ao desenvolvimento da síndrome de Reye ${ }^{7}$. Porém, o risco de síndrome de Reye é desconhecido através de salicilatos no leite materno $^{8}$.

\section{Considerações finais}

As evidências encontradas na literatura apontam que a maioria dos medicamentos são considerados seguros durante a amamentação entre eles cefalexina, ibuprofeno, paracetamol, fluoxetina, loratadina e hidroclorotiazida.

São contraindicados durante o período de amamentação bromocriptina, dissulfiram, anastrozol, amiodarona e os medicamentos antineoplásicos. Recomenda-se selecionar, sempre que possível, fármacos já estudados, que sejam minimamente excretados no leite materno e que não apresentam o risco de ocorrência de efeitos ad- 


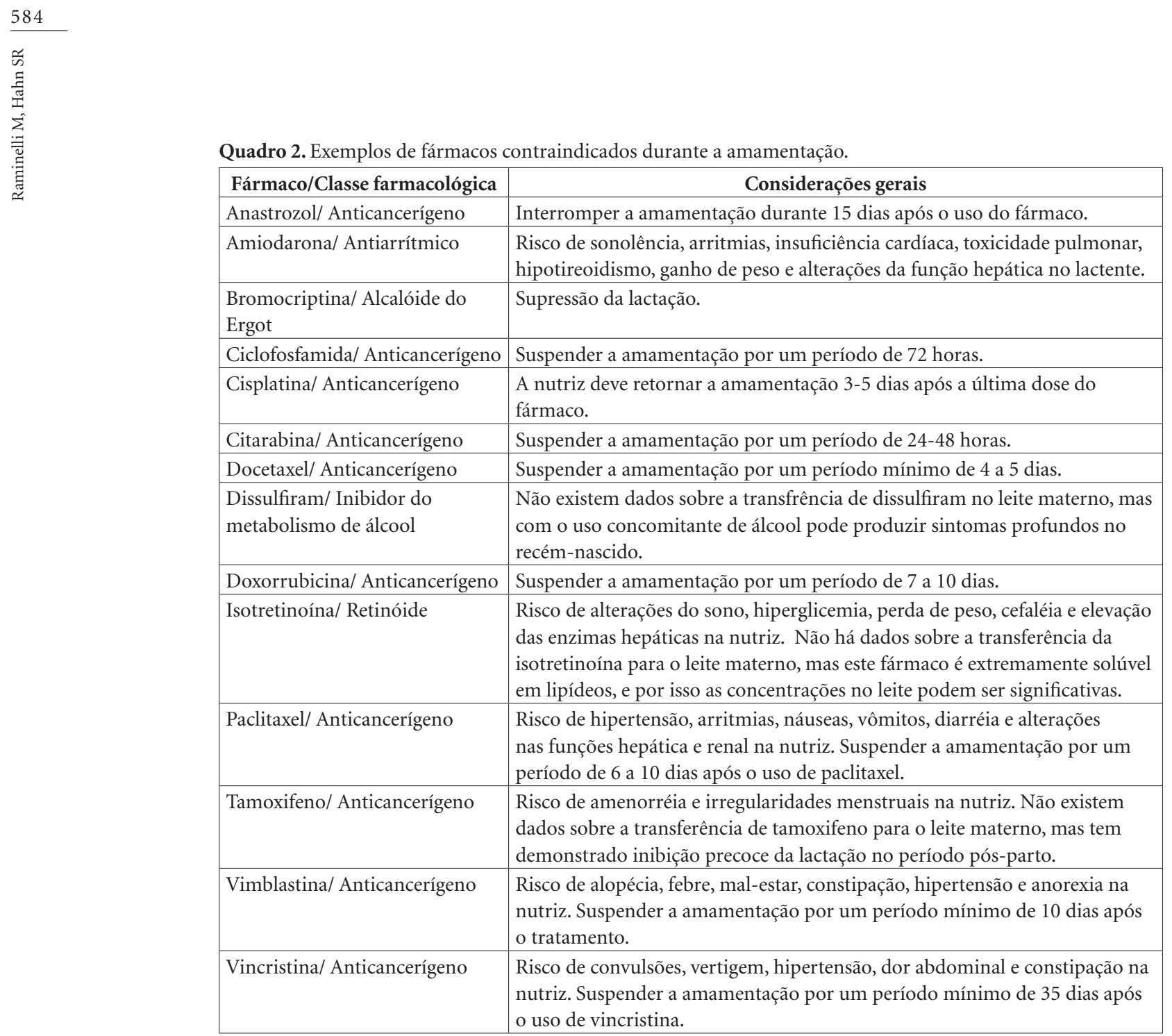

Adaptado de Hale e Rowe?

versos no lactente. Além disso, aspectos importantes devem ser considerados, tais como a toxicidade do fármaco, a condição de saúde e a idade do lactente, assim como a duração do tratamento farmacológico.

A importância e as vantagens do aleitamento materno para a mãe e o lactente são bem evidenciadas. O princípio primordial no uso de medicamentos para nutrizes baseia-se especialmente no conceito risco versus benefício.

O elo entre o uso de fármacos pela nutriz e a interrupção precoce do aleitamento materno, demonstra a real necessidade de atualização constante sobre a segurança dos fármacos utilizados na amamentação, servindo como apoio à prática clínica na orientação às nutrizes sobre o uso seguro desses medicamentos. Assim, a amamentação somente deverá ser interrompida diante de informações baseadas em evidências de que o fármaco utilizado pela nutriz cause efeitos negativos para o recém-nascido.

Dessa maneira, fazem-se necessários maiores investimentos em pesquisas que determinem o grau de segurança dos medicamentos durante a lactação, demonstrando as consequências clínicas da exposição do lactente aos fármacos presentes no leite materno.

\section{Colaboradores}

M Raminelli trabalhou na concepção e na redação do artigo e SR Hahn contribuiu na revisão crítica do conteúdo e na aprovação final da versão a ser publicada. 


\section{Referências}

1. World Health Organization (WHO). Exclusive breastfeeding. 2016 [acessado 2016 Abr 4]. Disponível em: http://www.who.int/nutrition/topics/exclusive_ breastfeeding/en/.

2. Victora CG, Bahl R, Barros AJD, França GVA, Horton S, Krasevec J, Murch S, Sankar MJ, Walker N, Rollins NC. Breastfeeding in the 21st century: epidemiology, mechanisms, and lifelong effect. Lancet 2016; 387(10017):475-490.

3. American Academy of Pediatrics, Section on Breastfeeding. Breastfeeding and the use of human milk. Pediatrics 2012; 129(3):e827-e841.

4. American Academy of Pediatrics. Committee on drugs. The transfer of drugs and other chemicals into human milk. Pediatrics 2001; 108(3):776-789.

5. Sachs HC; American Academy of Pediatrics, Committee on Drugs. The transfer of drugs and therapeutics into human breast milk: An update on selected topics. Pediatrics 2013; 132(3):e796-e809.

6. Hutchinson S, Marmura MJ, Calhoun A, Lucas S, Silberstein S, Peterlin BL. Use of Common Migraine Treatments in Breast-Feeding Women: A Summary of Recommendations. Headache 2013; 53(4):614-627.

7. Hale TW, Rowe HE. Medications and Mothers' Milk. $17^{\text {th }}$ ed. Amarillo: Hale Publishing LP; 2014.

8. National Institutes of Health. US National Library of Medicine. Drugs and Lactation Database (LactMed). 2016 [acessado 2016 Jun 5]. Disponível em: http:// toxnet.nlm.nih.gov/newtoxnet/lactmed.htm.

9. Lamounier JA, Chaves RG, Vieira GO. Medicamentos e Amamentação. In: Campos Júnior D, Burns DAR, organizadores. Tratado de pediatria: Sociedade Brasileira de Pediatria. $3^{a}$ ed. Barueri, São Paulo: Manole; 2014. p. 525-533.

10. Amir LH, Pirotta MV, Raval M. Breastfeeding - Evidence based guidelines for the use of medicines. Aust Fam Physician 2011; 40(9):684-690.

11. Mota LS, Chaves EMC, Barbosa RCM, Amaral JF, Farias LM, Almeida PC. Uso de medicamentos durante a lactação por usuárias de uma unidade básica de saúde. Rev Rene 2013; 14(1):139-147.

12. Fragoso VMS, Silva ED, Mota JM. Lactantes em tratamento medicamentoso da rede pública de saúde. Rev Bras Prom Saúde 2014; 27(2):283-290.

13. Chaves RG, Lamounier JA, César CC. Association between duration of breastfeeding and drug therapy. Asian Pac J Trop Disease 2011; 1(3):216-221.

14. Odom EC, Li R, Scanlon KS, Perrine CG, Grummer-Strawn L. Reasons for earlier than desired cessation of breastfeeding. Pediatrics 2013; 131(3):e726-e732.

15. Olang B, Heidarzadeh A, Strandvik B, Yngve A. Reasons given by mothers for discontinuing breastfeeding in Iran. Int Breastfeed J 2012; 7(1):1-7.

16. Boix-Amorós A, Collado MC, Mira A. Relationship between Milk Microbiota, Bacterial Load, Macronutrients, and Human Cells during Lactation. Front Microbiol 2016; 7:492.

17. Michaelsen KF. 2.1 Breastfeeding. World Rev Nutr Diet 2015; 113:92-96

18. Venancio SI, Escuder MML, Saldiva SRDM, Giugliani ERJ. Breastfeeding practice in the Brazilian capital cities and the Federal District: current status and advances. J Pediatr 2010; 86(4):317-324.
19. World Health Organization (WHO). Early initiation of breastfeeding. 2016 [acessado 2016 Abr 20]. Disponível em: http://www.who.int/elena/titles/early_breastfeeding/en/.

20. La Leche League International. What is colostrum? How does it benefit my baby? 2016. [acessado 2016 Abr 20]. Disponível em: www.llli.org/FAQ/colostrum. html.

21. Nice FJ, Luo AC. Medications and breast-feeding: Current concepts. J Am Pharm Assoc 2012; 52(1):8694.

22. Bowatte G, Tham R, Allen KJ, Tan DJ, Lau MXZ, Dai $\mathrm{X}$, Lodge CJ. Breastfeeding and childhood acute otitis media: a systematic review and meta-analysis. Acta Paediatr 2015; 104(467):85-95.

23. Lodge CJ, Tan DJ, Lau MXZ, Dai X, Tham R, Lowe AJ, Bowatte G, Allen KJ, Dharmage SC. Breastfeeding and asthma and allergies: a systematic review and meta-analysis. Acta Paediatr 2015; 104(467):38-53.

24. Peres KG, Cascaes AM, Nascimento GG, Victora CG Effect of breastfeeding on malocclusions: a systematic review and meta-analysis. Acta Paediatr 2015; 104(467):54-61.

25. Tham R, Bowatte G, Dharmage SC, Tan DJ, Lau MXZ, Dai X, Allen KJ, Lodge CJ. Breastfeeding and the risk of dental caries: a systematic review and meta-analysis. Acta Paediatr 2015; 104(467):62-84.

26. Horta BL, Loret de Mola C, Victora CG. Long-term consequences of breastfeeding on cholesterol, obesity, systolic blood pressure, and type-2 diabetes: systematic review and meta-analysis. Acta Paediatr 2015; 104(467):30-37.

27. Horta BL, Loret de Mola C, Victora CG. Breastfeeding and intelligence: systematic review and meta-analysis. Acta Paediatr 2015; 104(467):14-19.

28. Victora CG, Horta BL, Loret de Mola C, Quevedo L, Pinheiro RT, Gigante DP, Gonçalves H, Barros FC. Association between breastfeeding and intelligence, educational attainment, and income at 30 years of age: a prospective birth cohort study from Brazil. Lancet Glob Health 2015; 3(4):e199-e205.

29. França-Botelho AC, Ferreira MC, França JL, França EL, Honório-França AC. Breastfeeding and its relationship with reduction of breast cancer: a review. Asian Pacific J Cancer Prev 2012; 13(11):5327-5332.

30. Chowdhury R, Sinha B, Sankar MJ, Taneja S, Bhandari N, Rollins N, Bahl R, Martines J. Breastfeeding and maternal health outcomes: a systematic review and meta-analysis. Acta Paediatr 2015; 104(467):96-113.

31. Rowe H, Baker T, Hale TW. Maternal Medication, Drug Use, and Breastfeeding. Child Adolesc Psychiatric Clin N Am 2015; 24(1):1-20.

32. Hale TW. Breastfeeding. Drug entry into Human Milk Infant Risk Center. Texas Tech University Health Sciences Center 2016. [acessado 2016 Maio 2]. Disponível em: http://www.infantrisk.com/content/ drug-entry-human-milk.

33. Burkey BW, Holmes AP. Evaluating Medication Use in Pregnancy and Lactation: What Every Pharmacist Should Know. J Pediatr Pharmacol Ther 2013;18(3):247-258.

34. Nordeng H, Havnen GC, Spigset O. Drug use and breastfeeding. Norwegian Med Assoc 2012; 132(9):1089-1093. 
35. Soussan C, Gouraud A, Portolan G, Jean-Pastor MJ, Pecriaux C, Montastruc JL, Damase-Michel C, Lacroix I. Drug-induced adverse reactions via breastfeeding: a descriptive study in the French Pharmacovigilance Database. Eur J Clin Pharmacol 2014; 70(11):13611366.

36. Anderson PO, Valdés V. Variation of milk intake over time: clinical and pharmacokinetic implications. Breastfeed Med 2015; 10(3):142-144.

37. Anderson PO, Manoguerra AS, Valdés V. A review of adverse reactions in infants from medications in breastmilk. Clin Pediatr 2015; 55(3):236-244.

38. Wannmacher L. Fármacos em gestação e lactação. In: Fuchs FD, Wannmacher L. Farmacologia clínica: fundamentos da terapêutica racional. $4^{\text {a }}$ ed. Rio de Janeiro: Guanabara Koogan; 2010. p. 1146-1159.

39. Hotham N, Hotham E. Drugs in breastfeeding. Aust Prescr 2015; 38(5):156-159.

40. Berlin CM, Briggs GG. Drugs and chemicals in human milk. Semin Fetal Neonatal Med 2005; 10(2):149-159.

41. Davanzo R, Dal Bo S, Bua J, Copertino M, Zanelli E, Matarazzo L. Antiepileptic drugs and breastfeeding. Ital J Pediatr 2013; 39:50.

42. Rainsford KD. Ibuprofen: pharmacology, efficacy and safety. Inflammopharmacology 2009; 17(6):275-342.

43. Bertino E, Varalda A, Di Nicola P, Coscia A, Occhi L, Vagliano L, Soldi A, Perathoner C. Drugs and breastfeeding: instructions for use. J Matern Fetal Neonatal Med 2012; 25(Supl. 4):78-80.

44. Begg EJ, Duffull SB, Hackett LP, Ilett KF. Studying drugs in human milk: time to unify the approach. $J$ Hum Lact 2002; 18(4):323-332.

45. Ito S. Drug therapy for breast-feeding women. $N$ Engl $J$ Med 2000; 343(2):118-126.

46. Zhang BZ, Zhang HY, Liu HH, Li HJ, Wang JS. Breastfeeding and maternal hypertension and diabetes: a population-based cross-sectional study. Breastfeed Med 2015; 10(3):163-167.

47. Ost L, Wettrell G, Björkhem I, Rane A. Prednisolone excretion in human milk. J Pediatr 1985; 106(6):10081011.

48. Benyamini L, Merlob P, Stahl B, Braunstein R, Bortnik $\mathrm{O}$, Bulkowstein M, Zimmerman D, Berkovitch M. The safety of amoxicillin/clavulanic acid and cefuroxime during lactation. Ther Drug Monit 2005; 27(4):499502.

49. Salman S, Davis TME, Page-Sharp M, Camara B, Oluwalana C, Bojang A, D’Alessandro U, Roca A. Pharmacokinetics of transfer of azithromycin into the breast milk of african mothers. Antimicrob Agents Chemother 2016; 60(3):1592-1599.

50. Giamarellou H, Kolokythas E, Petrikkos G, Gazis J, Aravantinos D, Sfikakis P. Pharmacokinetics of three newer quinolones in pregnant and lactating women. Am J Med 1989;87(5A):49S-51S.

51. Kafetzis DA, Siafas CA, Georgakopoulos PA, Papadatos CJ. Passage of cephalosporins and amoxicillin into the breast milk. Acta Paediatr Scand 1981; 70(3):285288.

52. Hirose M, Hara Y, Hosokawa T, Tanaka Y. The effect of postoperative analgesia with continuous epidural bupivacaine after cesarean section on the amount of breastfeeding and infant weight gain. Anesth Analg 1996; 82(6):1166-1169.
53. Sammour RN, Ohel G, Cohen M, Gonen R. Oral naproxen versus oral tramadol for analgesia after cesarean delivery. Int J Gynaecol Obstet 2011; 113(2):144-147.

54. Rigourd V, de Villepin B, Amirouche A, Bruneau A, Seraissol P, Florent A, Urien S, Magny JF, Serreau R. Ibuprofen concentrations in human mature milk-first data about pharmacokinetics study in breast milk with AOR-10127 «Antalait» study. Ther Drug Monit 2014; 36(5):590-596.

55. Gardiner SJ, Doogue MP, Zhang M, Begg EJ. Quantification of infant exposure to celecoxib through breast milk. Br J Clin Pharmacol 2006; 61(1):101-104.

56. Jamali F, Keshavarz E. Salicylate excretion in breast milk. Int J Pharm 1981; 8(4):285-290.

57. Zylber-Katz E, Linder N, Granit L, Levy M. Excretion of dipyrone metabolites in human breast milk. Eur J Clin Pharmacol 1986; 30(3):359-361.

58. Notarianni LJ, Oldham HG, Bennett PN. Passage of paracetamol into breast milk and its subsequent metabolism by the neonate. Br J Clin Pharmacol 1987; 24(1):63-67.

59. Ilett KF, Paech MJ, Page-Sharp M, Sy SK, Kristensen JH, Goy R, Chua S, Christmas T, Scott KL. Use of a sparse sampling study design to assess transfer of tramadol and its O-desmethyl metabolite into transitional breast milk. $\mathrm{Br} J$ Clin Pharmacol 2008; 65(5):661-666.

60. Kelly LE, Chaudhry SA, Rieder MJ, 't Jong G, Moretti ME, Lausman A, Ross C, Berger H, Carleton B, Hayden MR, Madadi P, Koren G. A clinical tool for reducing central nervous system depression among neonates exposed to codeine through breast milk. Plos One 2013; 8(7):1-6.

61. Epperson CN, Jatlow PI, Czarkowski K, Anderson GM. Maternal fluoxetine treatment in the postpartum period: effects on platelet serotonin and plasma drug levels in breastfeeding mother-infant pairs. Pediatrics 2003; 112(5):e425-e429.

62. Merlob P, Stahl B, Sulkes J. Paroxetine during breast-feeding: infant weight gain and maternal adherence to counsel. Eur J Pediatr 2004; 163(3):135139.

63. Stowe ZN, Hostetter AL, Owens MJ, Ritchie JC, Sternberg K, Cohen LS, Nemeroff CB. The pharmacokinetics of sertraline excretion into human breast milk: determinants of infant serum concentrations. J Clin Psychiatry 2003; 64(1):73-80.

64. Rampono J, Hackett LP, Kristensen JH, Kohan R, Page-Sharp M, Ilett KF. Transfer of escitalopram and its metabolite demethylescitalopram into breastmilk. Br J Clin Pharmacol 2006; 62(3):316-322.

65. Brixen-Rasmussen L, Halgrener J, Jorgensen A. Amitriptyline and nortriptyline excretion in human breastmilk. Psychopharmacology 1982; 76(1):94-95.

66. Moretti ME, Ito S, Koren G. Disposition of maternal ketoconazole in breast milk. Am J Obstet Gynecol 1995; 173(5):1625-1626.

67. Moorhead AM, Amir LH, O'Brien PW, Wong S. A prospective study of fluconazole treatment for breast and nipple thrush. Breastfeed Rev 2011; 19(3):25-29.

68. Oo CY, Kuhn RJ, Desai N, Wright CE, McNamara PJ. Pharmacokinetics in lactating women: prediction of alprazolam transfer into milk. $\mathrm{Br} J$ Clin Pharmacol 1995; 40(3):231-236. 
69. Dusci LJ, Good SM, Hall RW, Ilett KF. Excretion of diazepam and its metabolites in human milk during withdrawal from combination high dose diazepam and oxazepam. Br J Clin Pharmacol 1990; 29(1):123126.

70. Söderman P, Matheson I. Clonazepam in breast milk. Eur J Pediatr 1988; 147(2):212-213.

71. Hilbert J, Radwanski E, Affrime MB, Perentesis G, Symchowicz S, Zampaglione N. Excretion of loratadine in human breast milk. J Clin Pharmacol 1988; 28(3):234-239.

72. Lucas Junior BD, Purdy CY, Scarim SK, Benjamin S, Abel SR, Hilleman DE. Terfenadine pharmacokinetics in breast milk in lactating women. Clin Pharmacol Ther 1995; 57(4):398-402.

73. Kaplan YC, Koren G, Ito S, Bozzo P. Fluconazole use during breastfeeding. Can Fam Physician 2015; 61(10):875-876.

74. Westermann L, Hügel R, Meier M, Weichenthal M, Zillikens D, Gläser R, Schmidt E. Glucocorticosteroid-resistant pemphigoid gestationis: successful treatment with adjuvant immunoadsorption. J Dermatol 2012; 39(2):168-171.

75. Boussageon R, Gueyffier F, Cornu C. Metformin as firstline treatment for type 2 diabetes: are we sure? BMJ 2016; 352:h6748.

76. Briggs GG, Ambrose PJ, Nageotte MP, Padilla G, Wan S. Excretion of metformin into breast milk and the effect on nursing infants. Obstet Gynecol 2005; 105(6):1437-1441.

77. Feig DS, Briggs GG, Kraemer JM, Ambrose PJ, Moskovitz DN, Nageotte M, Donat DJ, Padilla G, Wan S, Klein J, Koren G. Transfer of glyburide and glipizide into breast milk. Diabetes Care 2005; 28(8):1851-1855.

78. Ringholm L, Mathiesen ER, Kelstrup L, Damm P. Managing type 1 diabetes mellitus in pregnancy-from planning to breastfeeding. Nat Rev Endocrinol 2012; 8(11):659-667.

79. Chiasse N, Shamir R, Berant M, Etzioni A. Insulin in human milk and the prevention of type 1 diabetes. Pediatr Diabetes 2001; 2(4):175-177.

80. Whitmore TJ, Trengove NJ, Graham DF, Hartmann PE. Analysis of insulin in human breast milk in mothers with type 1 and type 2 diabetes mellitus. Int J Endocrinol 2012; 2012:1-9.

81. World Health Organization (WHO). Guidelines on HIV and infant feeding. Principles and recommendations for infant feeding in the context of HIV and a summary of evidence. WHO; 2010. [acessado 2016 Ago 9]. Disponível em: http://www.who.int/maternal_child_ adolescent/documents/9789241599535/en/

82. Brasil. Ministério da Saúde (MS). Departamento de DST, AIDS e Hepatites Virais. Capítulo 2. Manejo de crianças nascidas de mães infectadas pelo HIV. Protocolo clínico e diretrizes terapêuticas para manejo da infecção pelo HIV em crianças e adolescentes. Atualizado em 17/07/2014. [acessado 2016 Ago 9]. Disponível em: http://www.aids.gov.br/pcdt/pediatrico/2.

83. Palma P. Human breast milk: is it the best milk to prevent HIV transmission? J Virus Erad 2016; 2(2):112113.
84. Kuhar DT, Henderson DK, Struble KA, Heneine W, Thomas V, Cheever LW, Gomaa A, Panlilio AL. Updated US Public Health Service guidelines for the management of occupational exposures to human immunodeficiency virus and recommendations for postexposure prophylaxis. Infect Control Hosp Epidemiol 2013; 34(9):875-892.

85. Palombi L, Pirillo MF, Andreotti M, Liotta G, Erba F, Sagno JB, Maulidi M, Ceffa S, Jere H, Marchei E, Pichini S, Galluzzo CM, Marazzi MC, Vella S, Giuliano M. Antiretroviral prophylaxis for breastfeeding transmission in Malawi: drug concentrations, virological efficacy and safety. Antivir Ther 2012; 17(8):1511-1519.

86. Shapiro RL, Hughes MD, Ogwu A, Kitch D, Lockman S, Moffat C, Makhema J, Moyo S, Thior I, McIntosh $\mathrm{K}$, van Widenfelt E, Leidner J, Powis K, Asmelash A, Tumbare E, Zwerski S, Sharma U, Handelsman E, Mburu K, Jayeoba O, Moko E, Souda S, Lubega E, Akhtar M, Wester C, Tuomola R, Snowden W, Martinez-Tristani M, Mazhani L, Essex M. Antiretroviral regimens in pregnancy and breastfeeding in Botswana. N Engl J Med 2010; 362(24):2282-2294.

87. Dryden-Peterson S, Shapiro RL, Hughes MD, Powis K, Ogwu A, Moffat C, Moyo S, Makhema J, Essex M, Lockman S. Increased risk of severe infant anemia after exposure to maternal HAART, Botswana. J Acquir Immune Defic Syndr 2011; 56(5):428-436.

88. US Food and Drug Administration. How to request Domperidone for gastrointestinal disorders. 2016 [acessado 2016 Jul 3]. Disponível em: http://www.fda.gov/ Drugs/DevelopmentApprovalProcess/HowDrugsareDevelopedandApproved/ApprovalApplications/InvestigationalNewDrugINDApplication/ucm368736. htm.

89. US Food and Drug Administration. FDA talk paper: FDA warns against women using unapproved drug, domperidone, to increase milk production. 2004 [acessado 2016 Jul 3]. Disponível em: http://www. fda.gov/drugs/drugsafety/informationbydrugclass/ ucm173886.htm.

90. Zuppa AA, Sindico P, Orchi C, Carducci C, Cardiello V, Romagnoli C, Catenazzi P. Safety and efficacy of galactogogues: substances that induce, maintain and increase breast milk production. J Pharm Pharm Sci 2010; 13(2):162-174.

91. Kauppila A, Arvela P, Koivisto M, Kivinen S, Ylikorkala O, Pelkonen O. Metoclopramide and breast feeding: transfer into milk and the newborn. Eur J Clin Pharmacol 1983; 25(6):819-823.

92. Silva LR. Farmacoterapia pediátrica. In: Silva P. Farmacologia. $8^{\text {a }}$ ed. Rio de Janeiro: Guanabara Koogan; 2010. p. 1164-1179.

93. AlSaad D, ElSalem S, Abdulrouf PV, Thomas B, Alsaad T, Ahmed A, AlHail M. A retrospective drug use evaluation of cabergoline for lactation inhibition at a tertiary care teaching hospital in Qatar. Ther Clin Risk Manag 2016; 12:155-160.

Artigo apresentado em 28/10/2016

Aprovado em 30/03/2017

Versão final apresentada em 01/04/2017 